\title{
Commentary: Therapeutic Potential of Targeting the Auto-Inhibition of ASIC1a for Neuroprotection Against Ischemic Brain Injury
}

\author{
Matthew William, Sejla Turnadzic and Xiang-Ping Chu* \\ Department of Biomedical Sciences, School of Medicine, University of Missouri-Kansas City, Kansas City, MO, United States
}

Keywords: acid-sensing ion channel, acidosis, neuroprotection, auto-inhibition, cerebral ischemia

A Commentary on

Disruption of Auto-Inhibition Underlies Conformational Signaling of ASIC1a to Induce Neuronal Necroptosis

by Wang, J. J., Liu, F., Yang, F., Wang, Y. Z., Qi, X., Li, Y., et al. (2020). Nat. Commun. 11 (1):475. doi: 10.1038/s41467-019-13873-0

OPEN ACCESS

Edited by:

Yasuo Mori,

Kyoto University, Japan

Reviewed by:

Lachlan Rash,

The University of Queensland,

Australia

Wei-Guang Li,

Shanghai Jiao Tong

University, China

*Correspondence:

Xiang-Ping Chu

chux@umkc.edu

Specialty section:

This article was submitted to

Pharmacology of lon Channels and

Channelopathies,

a section of the journal

Frontiers in Pharmacology

Received: 10 September 2020

Accepted: 08 October 2020

Published: 24 November 2020

Citation:

William M, Turnadzic S, Chu X-P (2020) Commentary: Therapeutic Potential of Targeting the Auto-Inhibition of ASIC1a for Neuroprotection Against Ischemic

Brain Injury.

Front. Pharmacol. 11:604892.

doi: 10.3389/fphar.2020.604892

\section{INTRODUCTION}

Strokes are the fifth leading cause of death and disability in the United States (Zhou et al., 2018). This hypoxic event forces surrounding tissue to switch to anaerobic glycolysis and produce lactic acid, leading to acidosis (Rehncrona, 1985). The primary ion channels responsible for sensing acidosis are acid-sensing ion channels (ASICs) (Waldmann et al., 1997). Studies have shown that ASICs are involved in both physiological and pathological conditions (Chu and Xiong, 2012; Kellenberger and Schild, 2015). Among ASICs, the ASIC1a subtype is most sensitive to acidosis; it has high expression in the brain, and activation reveals calcium permeability (Xiong et al., 2004; Chu et al., 2014; Gründer and Pusch, 2015). We have shown that activation of ASIC1a during brain ischemia triggers neuronal injury, and deletion of ASIC1a generates neuroprotection (Xiong et al., 2004). Activation of ASIC1a also induces membrane depolarization (Jiang et al., 2009), subsequently leading to activation of other ion channels and receptors such as the N-methyl-D-aspartate (NMDA) receptor. Thus, ASIC1aNMDA receptor interaction contributes to acidosis-mediated ischemic brain injury (Gao et al., 2005; Isaev et al., 2008). Recently, studies from the Xu laboratory have demonstrated an additional mechanism by which acidosis could induce neuronal necroptosis through activation of ASIC1a, one independent of its ion-conducting function (Wang et al., 2015). They reported that acidosis caused receptor-interacting serine/threonine-protein kinase 1 (RIPK1) to interact with the C terminus (CT) "death motif" of ASIC1a protein, triggering phosphorylation of RIPK1 and resulting in neuronal necroptosis, independent of intracellular calcium levels. This demonstrated the crucial involvement of ASICla-RIPK1 activation in ischemic brain injury.

\section{ASIC1A AUTO-INHIBITION PREVENTS NEURONAL NECROPTOSIS}

A recent study reported in the Nature Communications (Wang et al., 2020) from the Xu laboratory expanded their previous findings and found that the cytoplasmic N terminus (NT) and CT of the ASICla protein 
interact in order to prevent necroptosis under normal physiological conditions. They showed that when an exposed derivative protein of the CT (known as CP-1-2) was released into cortical neurons, it recruited RIPK1 and induced necroptosis, even at physiological $\mathrm{pH}$. They hypothesized that the CT of ASICla binds to the NT at physiological $\mathrm{pH}$, preventing it from leading to necroptosis, like CP-1-2 did. Using the Förster resonance energy transfer technique, they observed the interactions between the CT and the NT and found that exposure to an acidic solution caused the masked NT and CT to separate. Returning to physiological $\mathrm{pH}$ of 7.4 reversed this separation. From this observation, they speculated that acidosis causes the CT and NT to unbind which lead to the binding of CT to RIPK1, resulting in necroptosis. To prove this prediction, they truncated the NT off in ASICla and found that neurons with this truncation underwent necroptosis at physiological $\mathrm{pH}$, further suggesting that the NT binds to the CT under non-acidic conditions, inhibiting necroptosis. Furthermore, they examined the free NT, which spontaneously binds to a protein known as N-ethylmaleimidesensitive fusion ATPase (NSF) during acidosis. They found that when NSF binds to the free NT, it prevents it from rebinding to the exposed CT, thus allowing the free CT to interact with and activate RIPK1, triggering necroptosis. To test this hypothesis, they used shRNA to knockdown the NSF protein and found that the neurons with less NSF expression revealed an attenuated acidosisinduced necroptosis. They also examined four negatively charged glutamate residues, which are able to bind to positively charged lysine residues on the $\mathrm{CT}$ death motif during physiological conditions, on the distal NT of ASICla protein. By mutating the glutamate residues to alanine, they fully removed all electrostatic interaction between the NT and CT. Freeing of these two ends resulted in increased levels of necroptosis. In order to prevent necroptosis during acidosis, they synthesized the peptide $\mathrm{NT}_{1-20}$, mimicking the NT of ASICla and found that when CP-1-2 was introduced into neurons, $\mathrm{NT}_{1-20}$ was able to bind to it, preventing RIPK1 activation, and blocking necroptosis. Pretreatment with the $\mathrm{NT}_{1-20}$ peptide before exposing ASIC1a to $\mathrm{pH} 6.0$ also significantly attenuated necroptosis. Finally, they injected the peptide $\mathrm{NT}_{1-20}$ into mouse lateral ventricles and found that it significantly reduced ischemic brain damage in an experimental stroke mouse model. In this studies, tags were added to the synthetic $\mathrm{NT}_{1-20}$ and CT ASIC peptides in order for them to get into to cytoplasm of the cells and have their effect. Collectively, their study suggests that synthetic peptides, such as $\mathrm{NT}_{1-20}$, may mimic auto-inhibition of ASIC1a, effectively attenuating acidosis-induced necroptosis during ischemic brain injury.

\section{DISCUSSION}

Although previous studies have examined how ASIC1a induces neuronal damage in stroke (Xiong et al., 2004; Gao et al., 2005; Zhou et al., 2019), multiple sclerosis (Vergo et al., 2011), and

\section{REFERENCES}

Belluzzi, E., Gonnelli, A., Cirnaru, M.-D., Marte, A., Plotegher, N., Russo, I., et al. (2016). LRRK2 phosphorylates pre-synaptic N-ethylmaleimide sensitive fusion spinal cord injury (Mazzone et al., 2017), this study from the $\mathrm{Xu}$ laboratory opens a new avenue to a potential therapeutic pathway by targeting ASIC1a auto-inhibition (Wang et al., 2020). Future studies are needed to investigate agents that could target the ASIC1a auto-inhibition pathway. One potential subject is the use of $\mathrm{NT}_{1-20}$ in preventing neuronal death. While $\mathrm{NT}_{1-20}$ seems like a promising peptide in preventing the CT from recruiting RIPK1, other functions of the peptide should also be examined. The NSF protein plays a critical role in fusing synaptic vesicles (Whiteheart et al., 1994; Belluzzi et al., 2016), so it is important to explore the consequences of inactivating it. Because knockdown of the NSF protein significantly reduced acidosis-induced necroptosis, it could be a potential therapeutic target against ischemic brain injury, but the consequences of inhibiting the NSF protein should be further investigated as it may interfere with synaptic vesicle fusion. RIPK1 is known to play a critical role in ASIC1a-mediated necroptosis by binding to the CT during ASIC1a activation (Wang et al., 2015). Selective inhibitor of RIPK1 such as necrostain-1 has been applied in stroke (Zhang et al., 2016), traumatic spinal cord injury (Wang et al., 2014), and amyotrophic lateral sclerosis (Re et al., 2014; Ito et al., 2016) and reveals protection in CNS diseases (Degterev et al., 2019; Yuan et al., 2019). The efficacy and selectivity of both direct ASIC inhibitors and RIPK1 inhibitors in preventing ASIC1a from committing acidosis-induced necroptosis should be studied carefully, as such inhibitors have had promising results in other pathways mediated by RIPK1 (Mifflin et al., 2020). ASIC1a plays an important role on its ion-conducting function such as synaptic plasticity, learning, and memory (Huang et al., 2015). Studies from the Xu laboratory uncovered an ion conduction-independent function of ASIC1a responsible for ischemic brain injury. Their study sheds new lights on potential therapeutic intervention of ASIC1amediated ischemic brain injury. Thus, targeting the autoinhibition of ASIC1a without affecting its physiological function becomes a desirable strategy in treatment of stroke patients.

\section{AUTHOR CONTRIBUTIONS}

All authors listed have made substantial, direct and intellectual contribution to the work, and approved it for publication.

\section{FUNDING}

The work was supported by grant from American Heart Association (19AIREA34470007) to XC.

(NSF) protein enhancing its ATPase activity and SNARE complex disassembling rate. Mol. Neurodegener. 11, 1. doi:10.1186/s13024-015-0066-z.

Chu, X.-P., Grasing, K. A., and Wang, J. Q. (2014). Acid-sensing ion channels contribute to neurotoxicity. Transl. Stroke Res. 5 (1), 69-78. doi:10.1007/ s12975-013-0305-y. 
Chu, X.-P., and Xiong, Z.-G. (2012). Physiological and pathological functions of acid-sensing ion channels in the central nervous system. Curr. Drug Targets. 13 (2), 263-271. doi:10.2174/138945012799201685.

Degterev, A., Ofengeim, D., and Yuan, J. (2019). Targeting RIPK1 for the treatment of human diseases. Proc. Natl. Acad. Sci. U.S.A. 116 (20), 9714-9722. doi:10. 1073/pnas.1901179116.

Gao, J., Duan, B., Wang, D.-G., Deng, X.-H., Zhang, G.-Y., Xu, L., et al. (2005). Coupling between NMDA receptor and acid-sensing ion channel contributes to ischemic neuronal death. Neuron 48 (4), 635-646. doi:10.1016/j.neuron.2005.10.011.

Gründer, S., and Pusch, M. (2015). Biophysical properties of acid-sensing ion channels (ASICs). Neuropharmacology 94, 9-18. doi:10.1016/j.neuropharm. 2014.12.016

Huang, Y., Jiang, N., Li, J., Ji, Y.-H., Xiong, Z.-G., and Zha, X.-m. (2015). Two aspects of ASIC function: synaptic plasticity and neuronal injury. Neuropharmacology 94, 42-48. doi:10.1016/j.neuropharm.2014.12.010.

Isaev, N. K., Stelmashook, E. V., Plotnikov, E. Y., Khryapenkova, T. G., Lozier, E. R., Doludin, Y. V., et al. (2008). Role of acidosis, NMDA receptors, and acidsensitive ion channel 1a (ASIC1a) in neuronal death induced by ischemia. Biochemistry (Mosc) 73 (11), 1171-1175. doi:10.1134/s0006297908110011.

Ito, Y., Ofengeim, D., Najafov, A., Das, S., Saberi, S., Li, Y., et al. (2016). RIPK1 mediates axonal degeneration by promoting inflammation and necroptosis in ALS. Science 353 (6299), 603-608. doi:10.1126/science.aaf6803.

Jiang, Q., Li, M.-H., Papasian, C. J., Branigan, D., Xiong, Z.-G., Wang, J. Q., et al. (2009). Characterization of acid-sensing ion channels in medium spiny neurons of mouse striatum. Neuroscience 162 (1), 55-66. doi:10.1016/j.neuroscience.2009.04.029.

Kellenberger, S., and Schild, L. (2015). International Union of Basic and Clinical Pharmacology. XCI. Structure, function, and pharmacology of acid-sensing ion channels and the epithelial Na+ channel. Pharmacol. Rev. 67 (1), 1-35. doi:10. 1124/pr.114.009225.

Mazzone, G. L., Veeraraghavan, P., Gonzalez-Inchauspe, C., Nistri, A., and Uchitel, O. D. (2017). ASIC channel inhibition enhances excitotoxic neuronal death in an in vitro model of spinal cord injury. Neuroscience 343, 398-410. doi:10.1016/ j.neuroscience.2016.12.008.

Mifflin, L., Ofengeim, D., and Yuan, J. (2020). Receptor-interacting protein kinase 1 (RIPK1) as a therapeutic target. Nat. Rev. Drug Discov. 19 (8), 553-571. doi:10. 1038/s41573-020-0071-y.

Re, D. B., Le Verche, V., Yu, C., Amoroso, M. W., Politi, K. A., Phani, S., et al. (2014). Necroptosis drives motor neuron death in models of both sporadic and familial ALS. Neuron 81 (5), 1001-1008. doi:10.1016/j.neuron.2014.01.011.

Rehncrona, S. (1985). Brain acidosis. Ann. Emerg. Med. 14 (8), 770-776. doi:10. 1016/s0196-0644(85)80055-x.

Vergo, S., Craner, M. J., Etzensperger, R., Attfield, K., Friese, M. A., Newcombe, J., et al. (2011). Acid-sensing ion channel 1 is involved in both axonal injury and demyelination in multiple sclerosis and its animal model. Brain 134 (Pt 2), 571-584. doi:10.1093/brain/awq337.
Waldmann, R., Champigny, G., Bassilana, F., Heurteaux, C., and Lazdunski, M (1997). A proton-gated cation channel involved in acid-sensing. Nature 386 (6621), 173-177. doi:10.1038/386173a0.

Wang, J.-J., Liu, F., Yang, F., Wang, Y.-Z., Qi, X., Li, Y., et al. (2020). Disruption of auto-inhibition underlies conformational signaling of ASIC1a to induce neuronal necroptosis. Nat. Commun. 11 (1), 475. doi:10.1038/s41467-019-13873-0.

Wang, Y., Wang, H., Tao, Y., Zhang, S., Wang, J., and Feng, X. (2014). Necroptosis inhibitor necrostatin-1 promotes cell protection and physiological function in traumatic spinal cord injury. Neuroscience 266, 91-101. doi:10.1016/j. neuroscience.2014.02.007.

Wang, Y. Z., Wang, J. J., Huang, Y., Liu, F., Zeng, W. Z., Li, Y., et al. (2015). Tissue acidosis induces neuronal necroptosis via ASIC1a channel independent of its ionic conduction. Elife 4, e05682. doi:10.7554/eLife.05682

Whiteheart, S. W., Rossnagel, K., Buhrow, S. A., Brunner, M., Jaenicke, R., and Rothman, J. E. (1994). N-ethylmaleimide-sensitive fusion protein: a trimeric ATPase whose hydrolysis of ATP is required for membrane fusion. J. Cell Biol. 126 (4), 945-954. doi:10.1083/jcb.126.4.945.

Xiong, Z.-G., Zhu, X.-M., Chu, X.-P., Minami, M., Hey, J., Wei, W.-L., et al. (2004). Neuroprotection in ischemia. Cell 118 (6), 687-698. doi:10.1016/j.cell.2004.08.026.

Yuan, J., Amin, P., and Ofengeim, D. (2019). Necroptosis and RIPK1-mediated neuroinflammation in CNS diseases. Nat. Rev. Neurosci. 20 (1), 19-33. doi:10. 1038/s41583-018-0093-1.

Zhang, S., Wang, Y., Li, D., Wu, J., Si, W., and Wu, Y. (2016). Necrostatin-1 attenuates inflammatory response and improves cognitive function in chronic ischemic stroke mice. Medicines 3 (3), 16. doi:10.3390/medicines3030016.

Zhou, R.-P., Leng, T.-D., Yang, T., Chen, F.-H., and Xiong, Z.-G. (2019). Acute ethanol exposure promotes autophagy-lysosome pathway-dependent ASICla protein degradation and protects against acidosis-induced neurotoxicity. Mol. Neurobiol. 56 (5), 3326-3340. doi:10.1007/s12035-018-1289-0.

Zhou, Z., Lu, J., Liu, W.-W., Manaenko, A., Hou, X., Mei, Q., et al. (2018). Advances in stroke pharmacology. Pharmacol. Ther. 191,23-42. doi:10.1016/j.pharmthera. 2018.05.012.

Conflict of Interest: The authors declare that the research was conducted in the absence of any commercial or financial relationships that could be construed as a potential conflict of interest.

Copyright (C) 2020 William, Turnadzic and Chu. This is an open-access article distributed under the terms of the Creative Commons Attribution License (CC BY). The use, distribution or reproduction in other forums is permitted, provided the original author(s) and the copyright owner(s) are credited and that the original publication in this journal is cited, in accordance with accepted academic practice. No use, distribution or reproduction is permitted which does not comply with these terms. 